\title{
Power Transformer Winding Fault Analysis using Transfer Function
}

\author{
Kalpana Patel, Student Member, IEEE, Narottam Das, Senior Member, IEEE, \\ A. Abu-siada, Senior Member, IEEE, and Syed Islam, Senior Member, IEEE \\ Department of Electrical and Computer Engineering, Curtin University, Perth, WA, Australia \\ e-mail: kalpana_patel800@yahoo.com.au,narottam.das@curtin.edu.au, a.abusiada@curtin.edu.au, and \\ s.islam@curtin.edu.au
}

\begin{abstract}
Condition monitoring of power transformers is essential to maintain the reliability of electricity grid. Frequency response analysis (FRA) is a useful tool for the detection of incipient mechanical faults within power transformers. There are various methods of evaluating the frequency spectrum for fault classification and quantification. This paper presents a useful method to detect and to identify various winding faults of a power transformer based on its FRA signature. Simulation results show that location of resonance frequencies which are the key parameters in identifying various winding deformations are uniquely altered due to different faults.
\end{abstract}

Index Terms - Frequency response analysis, power transformer, winding fault.

\section{INTRODUCTION}

F requency response analysis (FRA) is a useful technique for condition monitoring of a power transformer. It measures the frequency response of passive elements (e.g. resistor, inductor, and capacitor) in a power transformer over a wide range of frequencies. The main purpose of this test is to diagnose any incipient mechanical fault within a power transformer [1]. Usually, the FRA signature of a power transformer is compared with a reference data set. The difference between the measured data and reference data set is used to analyze the type and to identify the location of the fault [2-4]. Faults such as short-circuited turns, mechanical damage to winding and core, and loose turns are detectable through the transfer function (TF) method [5-6].

Each transformer has its own signature, however a transformer that does not have historical signature, a signature of other transformer of the same type and size may be considered for comparison. Short circuit faults produce forces that may cause winding deformation and winding movement that may lead to subsequent damage to the transformer. Therefore, it is essential to detect minor deformation and take a remedial action as soon as possible [7-12].

In this paper, a transformer model is proposed based on the TF approach, which can detect the winding's buckling fault, winding displacement and short circuit fault, etc. For this modelling, the ANSYS/ANSOFT Simplorer simulation tool is used to simulate various faults that in-service power transformer may experience. These results contributes to enrich the ability of the TF approach is an excellent useful technique for condition monitoring and engineering asset management to identify faults within power transformers.

This paper is organized as follows: the proposed power transformer model is given in section II, the power transformer fault analysis technique is elaborated in section III. Section IV presents the simulation results and discussion. Finally, the conclusion is drawn in section V.

\section{POWER TRANSFORMER MODEL}

A power transformer winding consists can be simulated using distributed elements of resistance, inductance and capacitance. Therefore, the TF approach can be used to identify winding deformation within the transformer. The TF is a mathematical representation of a system which is defined as the ratio of output to the input linear time-invariant system with zero initial conditions.

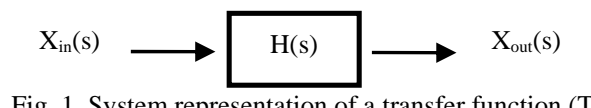

Fig. 1. System representation of a transfer function (TF).

Fig. 1 shows a system block diagram of a TF, where $\boldsymbol{X}_{\text {in }}(\mathrm{s})$ is the input signal, $\boldsymbol{X}_{\text {out }}(\mathrm{s})$ is the output signal and $\boldsymbol{H}(\mathrm{s})$ is the TF of the system; all in S-(Laplace transform) domain. The mathematical representation of the TF can be written as,

$$
H(s)=\frac{X_{\text {out }}(s)}{X_{\text {in }}(s)}
$$

The physical interpretation of the TF depends on the system parameters regardless the input and output signals. It also represents the variation of both the magnitude and phase angle with respect to the frequency.

The TF of a transformer is analyzed and the changes in inductance and capacitance or both are shown as either a shift of an existing, or creation of a new resonant frequency. This method relies on the principle that every transformer has a distinctive transfer function, commonly known as a "fingerprint". The capacitive and inductive components between the winding, core and tank of a transformer result in a frequency response that is unique to each individual unit.

For analysis, several fault cases involving windings in high voltage (HV) and low voltage (LV) side parameter variations are taken into account [3]. 


\section{POWER TRANSFORMER’s FAULT ANALYSIS TECHNIQUE}

Figs. 2(a) and 2(b) show the FRA amplitude and phase spectrum of a single disk in the HV side before and after short circuit fault.

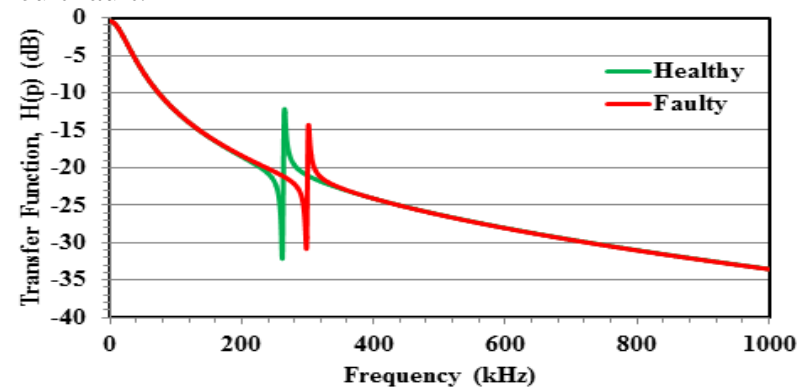

(a)

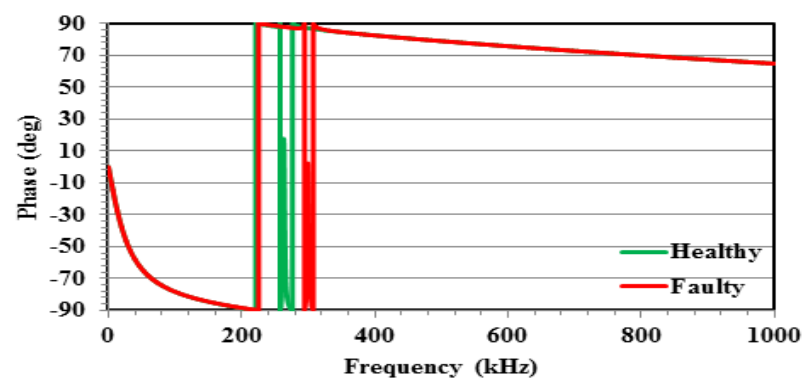

(b)

Fig. 2. (a) Transfer function, and (b) phase angle signature comparison of a healthy and faulty transformer for a single disk.

Fig. 2 (a) shows the magnitude of the TF that is due to fault, both location and peak amplitude of resonance frequency will be altered. Fig. 2(b) shows that the phase changes from $+90^{\circ}$ to $-90^{\circ}$ and the variations are mostly observed in the lower frequency bands. The phase variation between $+90^{\circ}$ and $-90^{\circ}$ is due to the inductive and capacitive components of the windings.

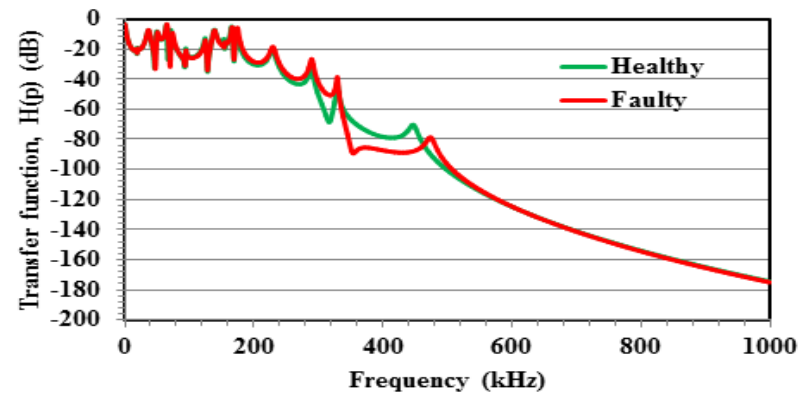

(a)

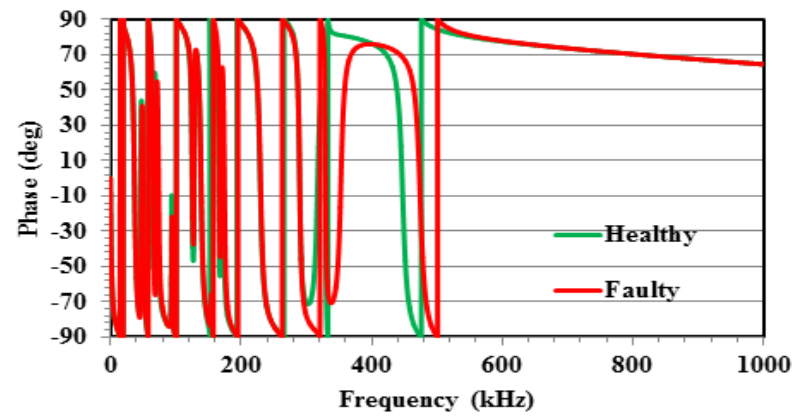

(b)

Fig. 3. (a) Transfer function, and (b) phase angle signature spectrum of a buckling fault of healthy and faulty transformer (10 disks).

\section{SimUlation RESUlts AND DisCUSSION}

Various faults are simulated by changing specific parameters and the TF plots for healthy and faulty signatures are compared $[11,12]$.

\section{Case\#1 Buckling Stress Fault}

Buckling stress is simulated by changing the value of shunt capacitor and series inductor on the LV winding side by $20 \%$, Fig. 3(a) shows the TF of healthy and faulty condition due to the buckling stress fault while Fig. 3(b) shows a comparison of the phase plot for buckling and healthy conditions. It is observed that the resonance frequencies are shifted towards the higher frequency due to the fault while there is a considerable change in the TF magnitude.

\section{Case\#2 Interdisk Fault}

According to [6], the inter-disk fault is simulated by short circuiting the series elements of the faulty turns.

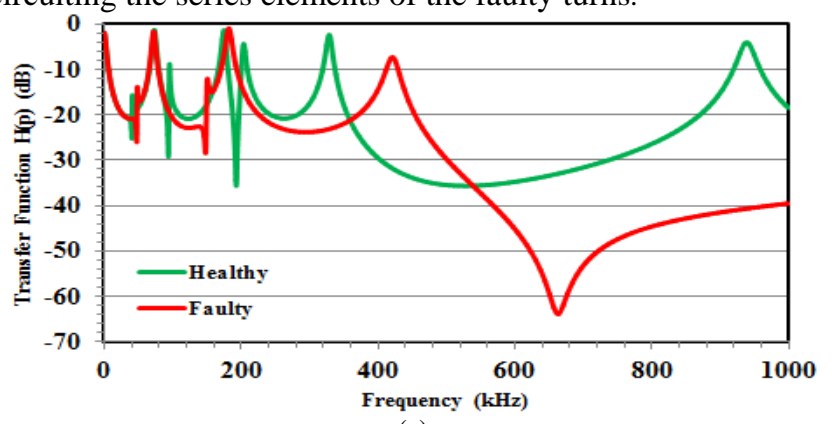

(a)

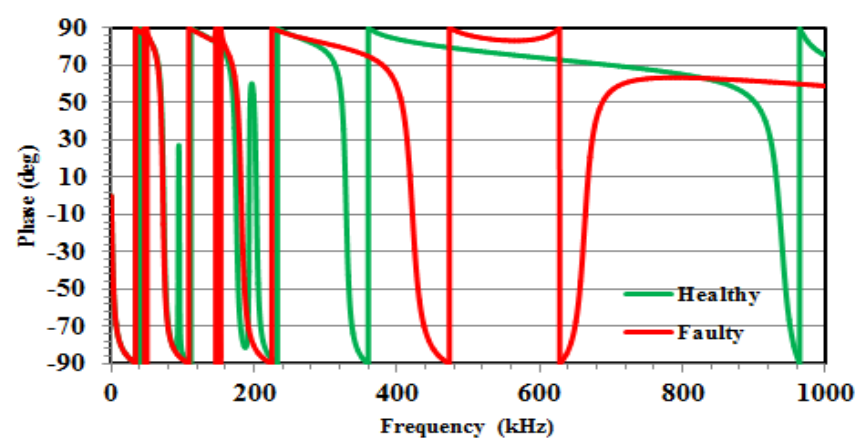

(b)

Fig. 4. (a) Transfer function, (b) phase angle signature spectrum of an interdisk fault (HV) of healthy and faulty transformer (5 disks).

Fig. 4shows the TF characteristics of a healthy and faulty transformer on the HV side and Fig. 4(b) shows the phase for the inter-disk fault. The figure shows a substantial change in the TF amplitude. Also, the resonance frequencies are shifted to the right side.

\section{Case\#3 Leakage Fault}

Leakage fault is simulated by increasing the value of the shunt conductor and shunt capacitor. [6].

For leakage fault, the TF characteristics pattern is shifted towards the lower frequency side with small change in frequency, as shown in Fig. 5. The change is more observable at the high frequency range which is attributed to the fact that at this range of frequency, capacitive components dominate the FRA characteristics. 


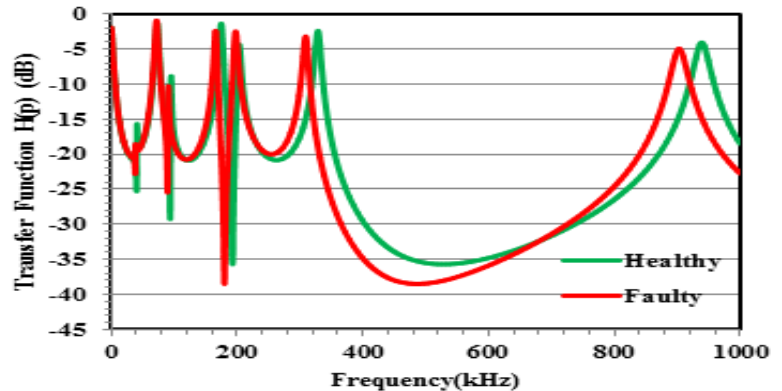

(a)

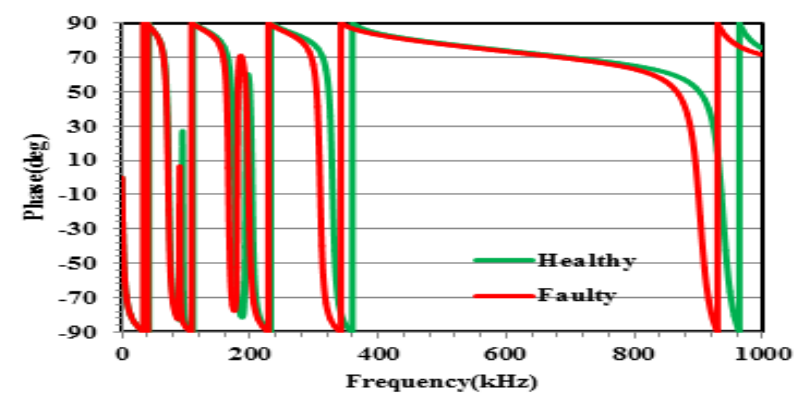

(b)

Fig. 5. (a) Transfer function, and (b) phase angle signature spectrum of a leakage fault (HV) of healthy and faulty transformer for 5 disks.

\section{Case\#4 Disk Space Variation}

This fault can be simulated by changing the series capacitor and mutual inductance between disks [5].

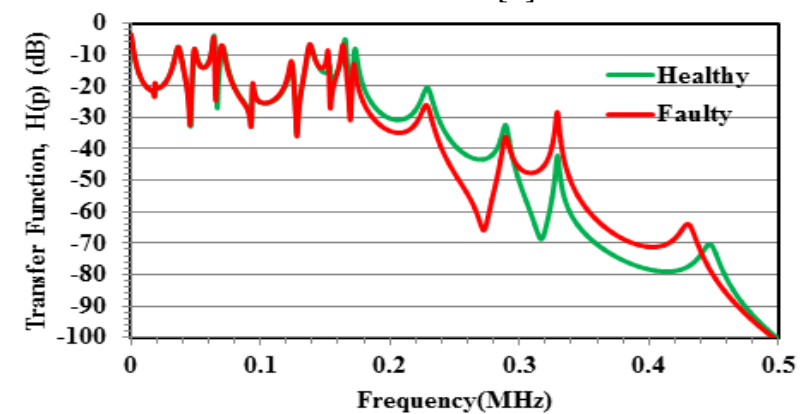

(a)

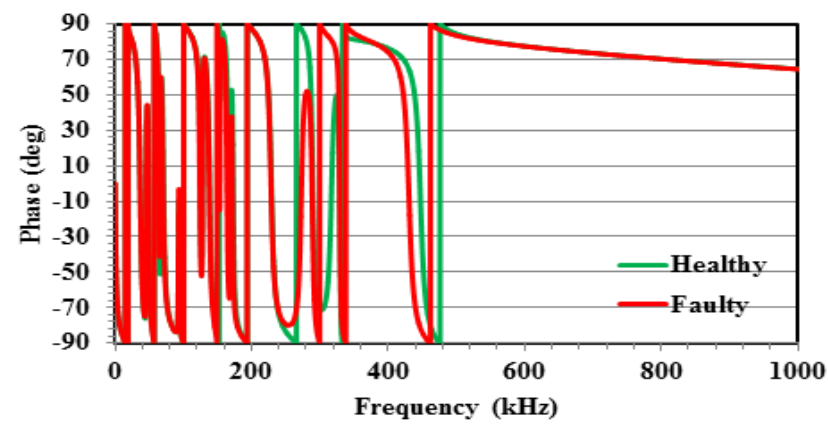

(b)

Fig. 6. (a) Transfer function and (b) phase angle signature spectrum of a disk space variation (HV) of healthy and faulty transformer for 10 disks.

Fig. 6 shows the TF characteristics for disk space variation fault. It is clear that the waveform remains unchanged for the low and medium frequency ranges. The resonance frequencies of the faulty signature are shifted toward the left side at high frequency range.

\section{CONCLUSION}

FRA is a useful diagnostic technique for detecting winding faults of a power transformer. The type and severity of the fault in a power transformer can be identified by analysing the FRA signature of the windings. Proper interpretation of FRA results assists in determining further action to be taken especially for the suspected or aged transformers and hence avoiding any possible catastrophic failures. The TF approach is a useful technique that can aid in constructing the FRA signature for a power transformer without the need to perform the practical test. Results from TF approach could be considered as a flag to take the transformer out of service to perform the practical FRA measurements. On the other hand, TF approach requires the electrical parameters of the transformer which is not available especially for old transformers to be known.

\section{ACKNOWLEDGEMENT}

This research is supported by the Centre for Smart Grid and Sustainable Power Systems, and the Faculty of Science and Enginering, Curtin University, Perth, Australia.

\section{REFERENCES}

[1] M. Wang and A. J. Vandermaar "Review of condition assessment of power transformers in service”, IEEE Electrical Insulation Magazine, vol. 18, no. 6, pp. 12-25, Nov./Dec. 2002.

[2] S. Islam, "Detection of shorted turns and winding movements in large power transformers using frequency response analysis”, IEEE PES winter meeting, vo1.3, pp. 2233-2238, Jan. 23-27, 2000, Singapore.

[3] S. A. Ryder, "Diagnosing Transformer Faults Using Frequency Response Analysis”, IEEE Electrical Insulation Magazine, vol.19, no.2, pp. 16-22, Mar./Apr. 2003.

[4] C. Bengtsson, "Status and trends in transformer monitoring". IEEE Trans. on Power Del. 11(3), 1379-1384, Jul. 1996.

[5] J. Bak-Jensen, B. Bak-Jensen, and S. D. Mikkelsen, "Detection of Faults and Aging Phenomena in Transformers by Transfer Functions", IEEE Trans. on Power Del., vol. 10, no. 1, Jan. 1999.

[6] S. Ryder, "Frequency analysis for diagnostic testing of power transformers,” Electricity Today, vol. 13, no. 6, pp.14-19, 2001.

[7] http://www.unistuttgart.de/ieh/forschung/veroeffentlichungen/2004_etg ___tenbohlen.pdf (Sighted on June 25, 2013).

[8] L. Coffeen, J. Britton, and J. Rickmann, "A new technique to detect winding displacements in power transformers using frequency response analysis,” in proc. of the 2003 IEEE Bologna Power Tech. Conf., June 23-26, Bologna, Italy, p. 7.

[9] E. P. Dick and C. C. Erven, "Transformer Diagnostic testing by Frequency Response Analysis,” IEEE Trans. on PAS, pp. 2144-2153, 1978.

[10] K. Jong-Wook, P. ByungKoo, J. Seung Cheol, K. Sang Woo, and P. PooGyeon, "Fault diagnosis of a power transformer using an improved frequency-response analysis," IEEE Transactions on Power Delivery,, vol. 20, pp. 169-178, 2005.

[11] S. Almas, T. Wenhu, and Q. H. Wu, "Modeling of a Power Transformer Winding for Deformation Detection Based on Frequency Response Analysis,” Chinese Control Conf. 2007 (CCC2007), July 26 31, 2007, pp. 506-510. Liverpool University, Liverpool, Hunan, UK.

[12] A. Reykherdt and A. Davydoy, "Case studies of factors influencing frequency response analysis measurements and power transformer diagnostics,” IEEE Electrical Insulation Magazine, vol. 27, no. 1, pp. 22-30, Jan./Feb. 2011. 